\title{
Comparison In Effect of Ocular Muscle Exercise With Conventional Treatment in Mechanical Neck Pain with Myopic Individuals
}

\author{
Dr.Poonam Rani, Dr.Deepak Raghav, Dr.Kopal Pajnee Dr. Humaira Khanam \\ ${ }^{I}$ (Santosh College Of Physiotherapy, Ghaziabad, Uttar Pradesh, India) \\ ${ }^{2}$ (Santosh Medical And Dental College,India) \\ ${ }_{3}^{3}$ (Santosh Medical And Dental College,Indi(A) ${ }^{4}$ lecturer In Sattam Bin Abdul Aziz University)
}

\begin{abstract}
To compare the effectiveness of conventional treatment with ocular muscle exercise in the treatment of mechanical neck pain in myopic individuals.

Methods: The Study included 14 young patients and were divided into two groups .Group A received conventional treatment(hot pack stretching,AROM and isometric exercises).Group B received ocular and conventional treatment(hot pack stretching,AROM and isometric exercises, ocular muscle exercises). All the outcome variables i.e. neck disability index.numerical pain rating scales, near point convergence were measured at 0 (pre-test), 7 sittinf and 15 sitting.

Results: The result of the study suggests the significant result within the groups after 3 week of treatment protocol.

Conclusion: The results of the study suggest that both the groups show improvement but ocular muscle exercises are beneficial for improving neck disablilit scales,numerical pain rating scales,near point convergence.
\end{abstract}

Keywords: cervical isometric exercises, Mechanical neck pain, myopia, NDI,. NPRS, NPC, ocular muscle exercise, . scalene .SCM, upper trapezius stretching.

\section{Introduction}

Neck pain is one of the most common complaints of musculoskeletal system. Neck pain commonly arises insidiously and is generally multifactorial in origin, including one or more of the following poor posture, anxiety, depression, neck strain and sporting or occupational activities. ${ }^{1}$ Spinal pain was most commonly reported as low back pain (43\%), closely followed by neck pain $(32 \%)$ and, far behind, by mid back pain $(13 \%)$.Approximately $2 / 3^{\text {rd }}$ of population at some point in their life experiences neck pain. . It is experienced by the people of all ages including children \& adolescent and is common in the adult general population, with typical 12-month prevalence estimate from $30 \%$ to $50 \%^{3}$.Prevalence rises with age in both sexes. In general, women suffer from complaints twice as often as men. ${ }^{2}$ Neck pain is multifactorial in its etiology and in its impact on the individual. The origin and exact pathphysiologic mechanisms of mechanical neck pain often remain obscure because trauma or severe degenerative conditions at working age are found only in a few cases. Excessive physical strain may cause micro trauma in connective tissues, and psychosocial stress may lead to increased muscular tension and pain. ${ }^{4}$ Several studies have reported that patients with neck pain also suffered from symptoms in the orofacial region, and headaches ${ }^{5} \mathrm{~A}$ large number research has been conducted to analyses the effect of ocular muscle exercise in myopic individual. But none of the studies have compared the effect of ocular muscle exercise with conventional treatment in mechanical neck pain between myopic and non myopic individual. Thus in this study it is intended to check the effect of ocular muscle exercise with conventional treatment in pain, functional outcome and near point convergence.purpose of this study is To compare the improvement in pain attained by the two-treatment approach conventional treatment and ocular muscle exercise by NPRS,To compare the improvement in neck disability attained by two-treatment approach conventional treatment and ocular muscle exercise by neck disability index scale,To compare the improvement in near point convergence attained by two-treatment approach conventional treatment and ocular muscle exercise by RAF rule.

\subsection{Subject's Criteria}

\section{Method}

The Study included 14 young patients with mechanical neck pain and myopic(-1.00 to -3.00 spherical dioptres) ,Pain persisting for 3 month difficulty in manging daily activities and studying.Participant who scored 6 to 17 on NDI. were divided into two groups . Each group was having seven subjects. the patient was collected from Santosh medical and dental college and hospital,Ghaziabad. Their ages ranged from 18-30 years old. 
Patients were informed that results drawn out of study will facilitate them to measure their performance and help in further enhancing the variable that improve their performance. A written consent form was taken from the patients who volunteered for the study and fulfilled the inclusion and exclusion criteria of the study.

Exclusion criteria of this study are Specific disorders of the cervical spine, such as disc prolapse, spinal stenosis.,Postoperative conditions in the neck and shoulder areas.,Spasmodic torticollis, migraine.,Hyper mobility syndrome.,Subjects with neurological deficit in upper limbs.History of fractures around cervical spine and shoulder area.,Shoulder diseases (tendonitis, bursitis, capsulitis),Inflammatory rheumatic diseases,Severe psychiatric illness and other diseases that prevent physical loading,Subject not on any medication for pain or myopia,Degenerative myopia,Distance visual acuity,Pseudomyopia

\section{2procedure}

Subjects were randomly selected for the study and by convenient sampling assigned to 2 different intervention groups. Each group was having 7 subjects. Group A received conventional treatment(hot pack stretching,AROM and isometric exercises).Group B received ocular and conventional treatment(hot pack stretching,AROM and isometric exercises, ocular muscle exercises). An informed consent was signed by all subjects before including in the study. All the subjects were assessed with NPRS, NDI and NPC test before giving treatment. After giving the treatment all subjects were reassessed and the data was subjected to statistical analysis.

\subsection{Outcome Measures}

Demographic variables of all subjects, such as age, eyesight, NDI scores were recorded. All subjects underwent a detailed orthopaedic assessment. A baseline measurement of dependent variables was taken on Numeric pain rating scale, Neck disability index, Near point convergence.

\section{4 treatment protocol}

\section{Treatment Protocol For Group A}

Isometric exercises,Patient position - sitting,Therapist position - standing Flexion- place hand on the forehead of patient ask patient to press forehead into palm and tell the patient to match your resistance. Avoid jerking of neck. Do 2 sets 12 repetition with 6sec hold. Extension - place hand on the back of head, ask the patient to press the back of the head into hands which are placed in the back near the top of the head. Side Bending - Place hand on the side of the head and ask the patient to press on hand \& attempt to side bend, trying to bring the ear towards the shoulder but not allowing motion. Rotation - The therapist place hand against the region just superior \& lateral to the eye \& patents attempt to turn the head to look over the shoulder but does not allow the motion. ${ }^{13}$ Stretching of Scalene :The patient lies supine with the head off the end of the plinth. Therapist stand at the head of treatment table and patient's head is supported by the therapist's right hand. Depress the clavicle and first rib with your left hand. Side bend the head and neck toward the right side. The neck is put in to extension. The face is rotated towards left side. ${ }^{56}$ Stretching of Sternocleidomastoid:With the patient supine, the therapist uses his or her left hand to press caudal through the lateral shoulder and clavicle .With the right hand, right side-bend and completely left rotate the patient's head and neck. Chin is tucked downward. ${ }^{105}$

\section{Treatment Protocol For Group B}

Isometric exercise,stretching of scalene, Stretching of Sternocleidomastoid,Ocular muscle exercises

Ocular Exercises-Palming exercise-the subject was asked to warm up the hands by rubbing the palms as follows. The base of the right pinkie will be on the base of the left pinkie making an upside down $\mathrm{v}$ with the palms. The subject was then asked to cover up the eyes with the palms. The base of the pinkie fingers will be right on the bridge of the nose (avoid applying pressure to the eyeballs.) if the subject is able to see the light through any of the holes between her fingers, she was asked to correct the placement of palms until she got to her eyes. The warmth of the cupped hand combined with blocking out all lights will relax a pair of tense eyeballs. The exercise was performed form sitting position on the school's chair and leaning forwards so that both elbows were rested on the school's desk.

Swinging exercise-this exercise works to counteract a frozen gaze and improve eyesight by bringing movement back to the process of seeing. each subject was asked to focus on a fixed object in front of her while she was in erect sitting position on the school chair then she was asked to swing her entire body totally first to right side then to the left side while she was keeping her eyes on the fixed object. the fixed object was a brightly yellow colored point drawn on the class black board that was two meters away from the chair and 1.15 meters height from the ground.

Centralization exercise (central fixation)-centralization is begin Fully present and aware in the moment. This exercise trains the eyes not to overstrain it by taking in too much at once it involves training the eyes to 
focus on a single point rather than an entire picture. Each subject was asked to look at the parts (part by part) of the colored picture that was placed on the class blackboard that was two meters away from the chair and 1.15 meters height from the ground. She was asked to name each part in the picture. The exercise was performed from erect sitting position on the school chair.

Turn and stretch the eyes far to the left and right. Continuing the movement back and forth from left to right., Turn the eyes upward, that is to say, look as far upward as possible without raising the head then without moving the head ,lower the eyes. looking as far down as possible .continue raising and lowering the eyes.raising the eyes ,look upward obliquely to the left-then lower them obliquely to the other side ,looking downward toward the right .raise the eyes upward obliquely to the right then stretch them obliquely downward to the left continue back and forth.Roll the eyes around in a circle, to the left upward, to the right downward, so on around then reverse, rolling them the other way around .continue until slightly tired.

Shut the eyes tightly and vigorously, squeezing the eyelids together as firmly as possible .open and repeat ten times,

focusing exercise for both eyes .look first at the point of a pencil held nearby, as in the upper photo, then shift to a distant cloud, or some tree or building on the horizon. Immediately upon seeing the distant object, shift back again to the pencil point.

(This is an exercise that should be performed without strain and at first with only two to four repetitions at a time. simply look cross-eyed as though trying to see the bridge of the nose with both eyes at once. eye exercise will applying for one minute and three repetitions with 3o seconds rest in-between with a total of nine minutes of training and four minutes rest in between. Therefore the exercise s perform from 15-30 minutes each day for each subject.)

(Protocol of group C and D is same as group A, B respectively)

\section{Data Analysis}

Data were summarized as Mean \pm SD. The effect of groups and periods on outcome measures were compared by repeated measures two factor analysis of variance (ANOVA) and the significance of mean difference within and between the groups was done by Tukey post hoc after ascertaining normality by ShapiroWilk test and homogeneity of variances by Levene's test .

\subsection{Group Allocation And Distribution Of Subjects}

\section{Result}

The present study compares the effect of ocular muscle exercise with conventional treatment in mechanical neck pain in myopic individuals. A total of 28 individuals aged 18-28 yrs either sex was recruited. The outcome measures of the study were neck disability index (NDI) score, numeric pain rating scale (NPRS) score and near point convergence (NPC) score observed at 0 sitting (pre treatment), and 7 sitting and 15 sitting (post treatment).

\begin{tabular}{|l|c|c|c|}
\hline Group & Subgroup & Group name & No. of subjects \\
\hline \multirow{2}{*}{ Myopic } & Conventional & Group A & 7 \\
\cline { 2 - 4 } & Ocular exercises + Conventional & Group B & 7 \\
\hline
\end{tabular}

\subsection{Basic Characteristics}

\subsection{Outcome Measures}

NDIThe pre (0 sitting) and post (7 sitting and 15 sitting) sitting neck disability index (NDI) scores of four groups

\begin{tabular}{|l|l|l|}
\hline Characteristics & $\begin{array}{l}\text { Group A } \\
(\mathrm{n}=7)\end{array}$ & $\begin{array}{l}\text { Group B } \\
(\mathrm{n}=7)\end{array}$ \\
\hline $\begin{array}{c}\text { Age (yrs): } \\
\text { Mean } \pm \text { SD }\end{array}$ & $23.14 \pm 3.13$ & $23.57 \pm 3.41$ \\
\hline
\end{tabular}

(Group A, Group B, Group C and Group D) are summarized in Table 3 and also shown graphically in

Fig. 3. Table 3 and Fig. 3 both showed that the mean NDI scores in all four groups decreased (improved) at post periods ( 7 sitting and 15 sitting) as compared to pre period ( 0 sitting) and the decrease (improvement) was evident highest in Group B followed by Group D, Group A and Group C, the least.

\begin{tabular}{|l|l|l|l|l|}
\hline Group & Pre sitting & Post sitting & \% change \\
\cline { 2 - 4 } & 0 Sitting & 7 Sitting & 15 Sitting & (0 Sitting to 15 Sitting) \\
\hline Group A & $16.43 \pm 3.95$ & $10.71 \pm 4.03$ & $6.14 \pm 3.72$ & $62.6 \%$ \\
\hline Group B & $16.57 \pm 4.28$ & $6.86 \pm 4.81$ & $4.14 \pm 2.61$ & $75.0 \%$ \\
\hline
\end{tabular}


Table 1: Pre and post sitting NDI scores (Mean $\pm \mathrm{SD}, \mathrm{n}=7$ ) of four groups

\begin{tabular}{|l|l|}
\hline F & pvalue \\
\hline value & 0.379 \\
& \\
\hline 1.07 & \\
\hline 2.41 & 0.92 \\
& \\
& \\
& \\
\hline
\end{tabular}

Table 2: Comparison of effect of groups and periods on NDI scores by repeated measures analysis of variance

\begin{tabular}{|l|l|l|}
\hline Comparisons & Group A & Group B \\
\hline 0 Sitting vs. 7 Sitting & 0.004 & $<0.001$ \\
\hline 0 Sitting vs. 15 Sitting & $<0.001$ & $<0.001$ \\
\hline 7 Sitting vs. 15 Sitting & 0.048 & 0.660 \\
\hline
\end{tabular}

Table 3: For each group, comparison (p value) of mean difference of NDI scores between the periods by Tukey NPRS post hoc test

The pre ( 0 sitting) and post (7 sitting and 15 sitting) sitting numeric pain rating scale (NPRS) scores of four groups (Group A, Group B, Group C and Group D) are summarized in Table 7 and also shown graphically in Fig. 7. Table 7 and Fig. 7 both showed that the mean NPRS scores in all four groups decreased (improved) at post periods ( 7 sitting and 15 sitting) as compared to pre period ( 0 sitting) and the decrease (improvement) was evident highest in Group B followed by Group D, Group A and Group C, the least

\begin{tabular}{|l|l|l|l|l|}
\hline \multirow{2}{*}{ Group } & Pre sitting & Post sitting & $\begin{array}{l}\text { \% change } \\
\text { (0 Sitting to 15 Sitting) }\end{array}$ \\
\cline { 2 - 4 } & 0 Sitting & 7 Sitting & 15 Sitting & $68.9 \%$ \\
\hline Group A & $6.43 \pm 0.98$ & $4.29 \pm 1.98$ & $2.00 \pm 1.00$ & $80.9 \%$ \\
\hline Group B & $6.71 \pm 1.60$ & $2.71 \pm 0.76$ & $1.29 \pm 0.49$ & 89 \\
\hline
\end{tabular}

Table 4: Pre and post sitting NPRS scores (Mean \pm SD, $n=7$ ) of four groups

\begin{tabular}{|l|l|}
\hline $\begin{array}{l}\text { F } \\
\text { value }\end{array}$ & $\begin{array}{l}\text { p } \\
\text { value }\end{array}$ \\
\hline 1.06 & 0.384 \\
& \\
\hline 133.32 & \\
\hline 1.29 & 0.282 \\
& \\
& \\
& \\
& \\
\hline
\end{tabular}

Table 5: Comparison of effect of groups and periods on NPRS scores by repeated measures analysis of variance (ANOVA)

\begin{tabular}{|l|l|l|}
\hline Comparisons & Group A & Group B \\
\hline 0 Sitting vs. 7 Sitting & 0.025 & $<0.001$ \\
\hline 0 Sitting vs. 15 Sitting & $<0.001$ & $<0.001$ \\
\hline 7 Sitting vs. 15 Sitting & 0.012 & 0.384 \\
\hline
\end{tabular}

Table 6: For each group, comparison ( $p$ value) of mean difference of NPRS scores between the periods by NPC

Tukey post hoc test 
The pre ( 0 sitting) and post (7 sitting and 15 sitting) sitting near point convergence (NPC) scores of four groups (Group A, Group B, Group C and Group D) are summarized in Table 11 and also shown graphically in Fig. 11. Table 11 and Fig. 11 both showed that the mean NPC scores in all four groups decreased (improved) at post periods ( 7 sitting and 15 sitting) as compared to pre period ( 0 sitting) and the decrease (improvement) was evident highest in Group B followed by Group D, Group A and Group C, the least.

\begin{tabular}{|l|l|l|l|l|}
\hline Group & Pre sitting & Post sitting & $\begin{array}{l}\text { \% change } \\
\text { (0 Sitting to 15 Sitting) }\end{array}$ \\
\cline { 2 - 4 } & 0 Sitting & 7 Sitting & 15 Sitting & $24.1 \%$ \\
\hline Group A & $15.73 \pm 5.69$ & $13.27 \pm 6.15$ & $11.94 \pm 5.63$ & $37.9 \%$ \\
\hline Group B & $15.81 \pm 6.45$ & $11.03 \pm 4.96$ & $9.83 \pm 5.58$ & \\
\hline
\end{tabular}

Table 7: Pre a post sitting NPCnd scores (Mean \pm SD, $n=7$ ) of four groups

\begin{tabular}{|c|c|}
\hline F & p \\
value & value \\
\hline 0.11 & 0.953 \\
& \\
\hline 36.91 & $<0.001$ \\
0.94 & 0.477 \\
& \\
& \\
& \\
& \\
\hline
\end{tabular}

Table 8: Comparison of effect of groups and periods on NPC scores by repeated measures analysis of variance (ANOVA)

\begin{tabular}{|l|l|l|}
\hline Comparisons & Group A & Group B \\
\hline 0 Sitting vs. 7 Sitting & 0.492 & 0.003 \\
\hline 0 Sitting vs. 15 Sitting & 0.038 & $<0.001$ \\
\hline 7 Sitting vs. 15 Sitting & 0.982 & 0.992 \\
\hline
\end{tabular}

Table 8.13: For each group, comparison ( $\mathrm{p}$ value) of mean difference of NPC scores between the periods by Tukey post hoc test

\section{Discussion}

The aim of this study to compare the effect of ocular muscle exercises and conventional treatment in mechanical neck pain individuals having myopia and without myopia. The result of the study suggest insignificance differences between groups and suggest the significant result within the groups,

On analysis within the groups for NDI, data was found to be significant in group A,B and insignificant in group C,D between period of 0-7 days, significant in group A , C , D and insignificant in group C in between7-15 days and significant in all four groups between period of 0-15 days. However improvement in NDI scores by $62.6 \%, 75.0 \%, 60.2 \%$ and $64.0 \%$ in was observed in Group A, Group B, Group C and Group D respectively with maximum improvement seen in group B and minimum improvement seen group C. On analysis within the group for NPRS, it was found to be significant in group A,B,C,D between period 0-7 days , significant in group A,C,D or insignificant in group B between period of 7-15 days and significant in all groups between period 0-15 days. However improvement in NPRS by $68.9 \%, 80.9 \%, 65.2 \%$ and $75.0 \%$ was observed in Group A, Group B, Group C and Group D respectively with maximum improvement was seen in-group B and minimum improvement seen in group C. On analysis within the groups for NPC, it was found to be significant in groups C,D or insignificant in groups A,B between period of 0-7 days, insignificant in all groups between period 7-15 days and significant in groups A,B,D or insignificant in group C between period 0-15 days however improvement in NPC by $24.1 \%, 37.9 \%, 20.5 \%$ and $31.6 \%$ was observed in Group A, Group B, Group C and Group D respectively with maximum improvement was seen in group B and minimum seen in group C.

In previously published studies on this subject indicating ability of conventional treatment to reduced pain and NDI .the neck disability index is a condition specific disability measure.Fritz and Brennan revealed that those receiving matched intervention demonstrated greater changes in both the NDI and pain rating score.NPRS improvement due to the activation of muscle mechanoreceptors and joint mechanoreceptors, during an isometric contraction leads to localized activation of the periaqueductal grey (PAG) matter in mid brain that plays a role in descending modulation of pain. Nociceptive inhibition then occurs at the dorsal horn of the spinal 
cord, as simultaneous gating takes place of nociceptive impulses in the dorsal horn, due to mechano-receptor stimulation. NDI improvement: Neck pain intensity/duration and disability due to neck pain generally showed moderate to good correlations.

Willford et al found that subject wearing multifocal lenses had a greater degree of forward head posture when compared with non -multifocal lenses wearer.Treleaven et al found postural stability and oculomotor control,such as altered smooth pursuit and saccadic eye movement can present in patients with neck disorders of either an insidious or traumatic nature as a result of cervical somatosensory dysfunction. An increased gain of the cervico-ocular reflex has also been demonstrated in patients with whiplash .Altered smooth pursuit neck torsion control occurs in neck pain subjects (both idiopathic and traumatic) but not in those with vestibular disorders and central nervous system dysfunction..

Chairy Wihlom found strong relationship between visual problem and pain in neck.A variation of oculomotor load was obtained by combining an alteration of visual distance and the application of minus lenses. Increases in electromyography (EMG) amplitudes were observed in the right frontalis, masseter, deltoid, middle trapezius, levator, and upper trapezius and upper neck muscles, including the splenius. A correlation between visual discomfort and pain in the neck and shoulder has been reported.

A number of previous studies which have shown that there has been effect of different eye exercise on refractive error. Koslowe et al and GilmartinB.there are very few studies which show effect of eye exercises on near point of convergence. scheiman $\mathrm{M}$ at .al and Hilreth $\mathrm{HR}$ et al. says that visual training improves near point of convergence. The reason through to be there behind effect of the eye exercises is, it strengthen accommodation, which is power of changing the focus of eyes for vision. The exercise is effective for long term benefits of the intervention. The greater effects of eye exercise were found in improvement of NPC in myopia. It may be due to the fact that in eye focusing exercise, the lenses convergence and relaxes, thus it affect the power of accommodation and with proper practice of eye exercise . it improves the accommodation power this would result in better improvement of NPC.

\section{Relevance To Clinical Practice}

Ocular muscle exercises shows better improvement in near point convergence and function and pain comparison to conventional treatment in females neck pain. So ocular muscle exercises can be used in. clinical practice in the treatment of chronic neck pain .The importance of the neck for postural stability, head and eye movement control, as well as the nature of the changes in sensorimotor control seen in those with neck disorders, assessment and management of such disturbances should form an important part of the multimodal approach to neck pain. This should include addressing the causes of the altered cervical somatosensory input such as improving neuromuscular function and decreasing pain and inflammation as well as a tailored sensorimotor exercise program to improve identified deficits in postural stability and head and eye movement control

\section{Conclusion}

In this study no effect of ocular muscle exercises were found on NDI, NPRS, NPC .Hence this study conclude by stating that null hypothesis accepted.

\section{VII1. limitations}

- The size of the sample was small.

- Duration of the study was limited.

- Procedural and instrumental error could not be controlled during the study.

\section{References}

[1]. Blinder AI.cervical spondylosis and neck pain BMJ 2007;334:527-531

[2]. Ruud Groeneweg \& Hans Kropman,:The effectiveness and cost-evaluation of manual therapy and physical therapy in patients with sub-acute and chronic nonspecific neck pain;BMC musculoskeletal disorders 2010, 11:14

[3]. Hogg-Johnson,Gabrielle van der Velde\& DC, Linda J. Carroll:The Burden and Determinants of Neck Pain in the general population;J. Euro Spine J (2008) 17 1: S39-S51.

[4]. Victoria Misailidou,Paraskevi Malliou, Anastasia Beneka\&Alexandros Karagiannidis:Assessment of patients with neck pain: a review of definitions, selection criteria, and measurement tool.

[5]. Roy La Touche, *y Ce’ sar Ferna' ndez-de-las-Peñ as,zx \{ Josue’ Ferna' ndez-Carnero,zx \{Santiago Di'az-Parreñ o, *y Alba ParisAlemany,*y and Lars Arendt-NielsenBilateral Mechanical-Pain Sensitivity Over the Trigeminal Region in Patients With Chronic Mechanical Neck Pain)

[6]. vandanaj.rathod,devanshi P.desai jagatheesan Alagesan:effect of eye exercise on myopia randomized controlled study (2011)

[7]. Vision Therapy-Based Program for Myopia Control in Adolescents Samia A. Abdel Rahman Mohamed(2013)390-396

[8]. wim jorritsma.grietje.E.de vries,Pieter u.dijkstra.janH.B.Geertzen.Michiel F.Reneman Neck pain and disability scale and neck disability index;validity of dutch language versions 2011 springlink.com

[9]. Samia A. Abdel Rahman Mohamed Vision Therapy-Based Program for Myopia Control in Adolescents Middle-East Journal of Scientific Research 13 (3): 390-396, 2013 
[10]. Jeremy A. Guggenheim,1 Kate Northstone,2 George McMahon,2 Andy R. Ness,3 Kevin Deere,4Calum Mattocks,5 Beate St Pourcain,2 and Cathy Williams2Time Outdoors and Physical Activity as Predictors ofIncident Myopia in Childhood: A Prospective Cohort StudyInvestigative Ophthalmology \& Visual Science, May 2012, Vol. 53, No. 6

[11]. by Clairy Wiholm, PhD,1 Hans Richter, PhD,2 Svend Erik Mathiassen, PhD,2 Allan Toomingas, PhD 3, 4 Associations between eyestrain and neck-shoulder symptoms among callcenter operators SJWEH Suppl 2007;(3):54-59

[12]. Julia Treleaven Sensorimotor disturbances in neck disorders affecting postural stability, head and eye movement control Manual Therapy $13(2008) 2-11$

[13]. Bexander CS1, Hodges PW. Cervico-ocular coordination during neck rotation is distorted in people with whiplash-associated disorders.

[14]. B. O. Dubrovsky And H. Barbas X Frontal Projections Of Dorsal Neck And Extraocular Muscles Experimental Neurology 55, 680693 (1977)

[15]. Cuccia A, Caradonna C. The relationship between the stomatognathic system and body posture. Clinics. 2009;64(1):61-6. 